\title{
Special issue on multidimensional signal processing applications
}

\author{
Rudolf Rabenstein • Jörg Velten
}

Published online: 1 January 2014

(C) Springer Science+Business Media New York 2013

This special issue on multidimensional signal processing applications covers a broad range of methodologies and algorithms for spatially extended configurations of sensors and emitters for various purposes and applications. The spectrum of the typical wavelengths for these applications ranges from very short ones in electron transmission microsopy to visibile light, up to radio frequencies and further to sound waves in the audio range suitable for human perception. In spite of the diversity of the underlying physical principles there are similarities in the arising problems. Consequently also some of the approaches to the processing of the resulting sensor or emitter signals are shared between these applications.

One common problem is the detection of drift and translation between images acquired by electron transmission microsopy and conventional cameras. Also the detection of motion in video sequences falls into this category. Another set of problems is concerned with the detection of the direction of a source of either electromagnetic or sound waves using suitable receiver arrays. But the situation may also be turned around by forming directed wave fronts from loudspeaker arrays to produce sound fields with certain spatial requirements.

This set of seven papers shows that modern multidimensional signal processing overcomes the traditional separation of image processing and audio processing. The presented methods and algorithms prescind from plain processing of available sensor or emitter signals. Instead the focus lies on physical processes and mathematical foundations underlying all applications.

The paper A comparison between minimum variance control and other online compensation methods for specimen drift in transmission electron microscopy by A. Tejada and A.J. den Dekker presents an online method to reduce image blurring by specimen drift dur-

\footnotetext{
R. Rabenstein $(\varangle)$

Chair of Multimedia Communications and Signal Processing, Friedrich-Alexander-University Erlangen-Nürnberg, Cauerstr. 7, 91058 Erlangen, Germany e-mail: rabenstein@LNT.de

J. Velten

Faculty of Electrical, Information and Media Engineering, University of Wuppertal, 42119 Wuppertal, Germany

e-mail: velten@uni-wuppertal.de
} 
ing exposure. They achieve this reduction by employing an adaptive control algorithm for horizontal and vertical drift.

A related problem for an application in image processing is discussed in Estimation of horizontal and vertical translations of large images based on columns and rows mean energy matching by E. Skubalska-Rafajlowicz. Here the 2-D translation problem is broken down to one dimension, resulting in computational savings for large images. The solution is reached by optimzation of a suitably defined energy measure.

Detecting translations between subsequent frames is also the topic of the paper $A$ multidimensional wave digital filter bank for video-based motion analysis by T. Schwertfeger, J. Velten, and A. Kummert. They solve the problem by space-time processing methods, i.e. by implementing fan-shaped transfer functions by multidimensional wave digital filters.

Space-time processing is also employed for shaping the directional characteristics of antennas in the radio frequency domain in Electronically scanned RF-to-bits beam aperture arrays using 2-D IIR spatially bandpass digital filters by A. Madanayake et al. The authors show a fixed-point implementation of a two-dimensional digital filter for processing radio frequencies in the $\mathrm{GHz}$ range.

Consideration of directional characteristics paves the way to localization of sources in the acoustic domain as well. The fundamental problems are similar as for radio frequencies, although frequency range and receiver technologies are quite different. A unifying perspective on acoustic source localization is offered by $\mathrm{P}$. Bestagini et al. in their paper TDOA-based acoustic source localization in the space-range reference frame. They start with time-differences-of-arrival detected from recorded source signals between different microphones and adopt a new reference frame by combining spatial coordinates and range differences.

The conversion from time differences to range differences requires knowledge of the propagation speed which depends on the medium in which waves travel. The paper Closedform estimation of the speed of propagating waves from time measurments by $\mathrm{P}$. Annibale and R. Rabenstein shows how the propagation speed can be estimated along with the source position from the same set of microphone signals.

Finally K. Helwani, S. Spors, and H. Buchner consider directional information for emitters of sound waves rather than sensors. In The synthesis of sound figures they show how to drive arrays of loudspeakers to create sound fields with spatially fixed zones of high sound pressure level along with other zones of low levels. The presented approach is based on a Green's function description of sound propagation.

We would like to thank all authors for their carefully prepared contributions, the reviewers for their valuable suggestions in improving the presentation, and the Editor-in Chief, Zhiping Lin, for his constant support and encouragement. 